Families expressing interest $(n=67)$

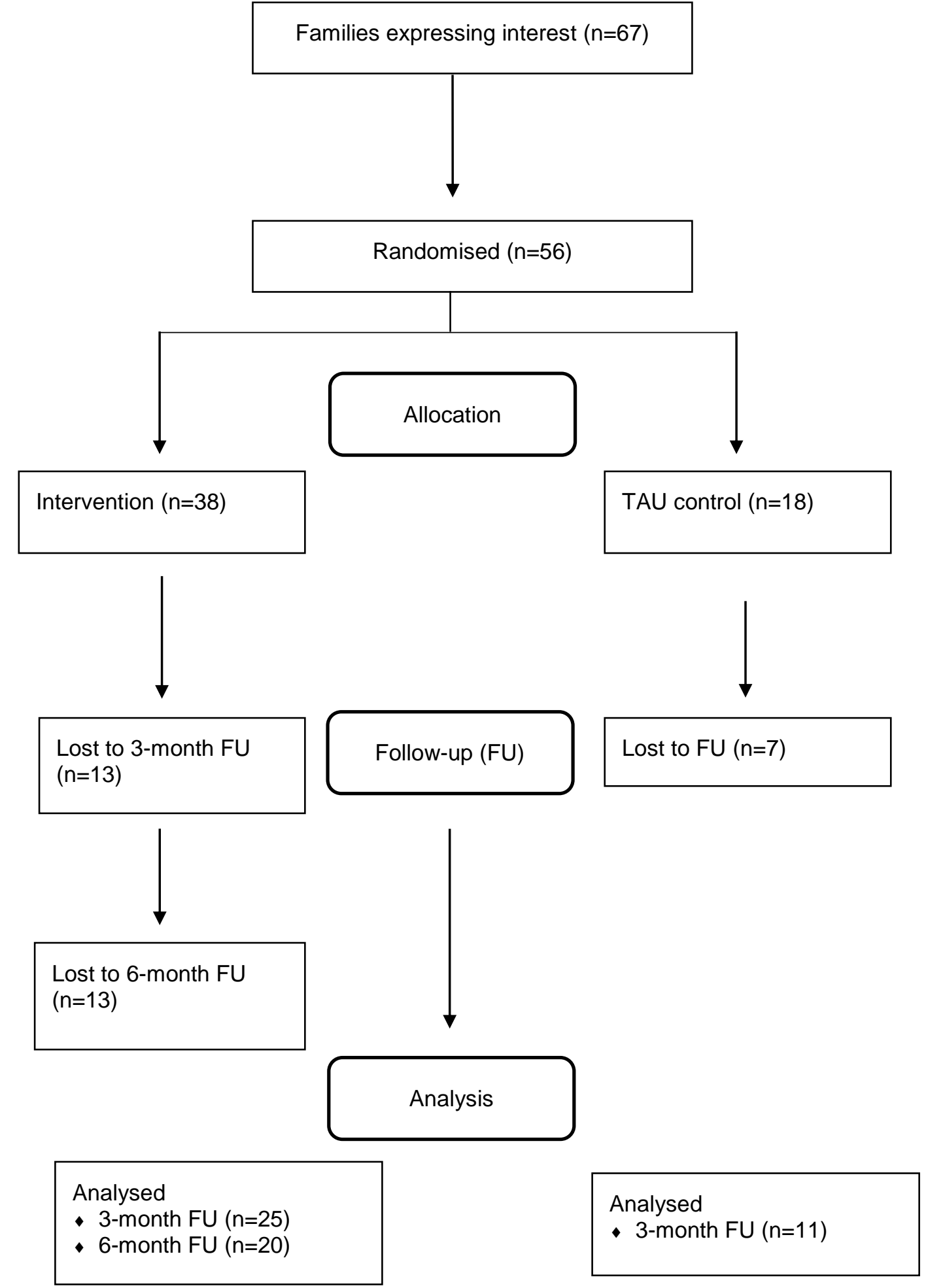

Figure 1. Participant flow diagram 


\section{Baseline Charactersitics}

Table 1. Participant baseline characteristics

\begin{tabular}{llll}
\hline Family characteristics & All $(\boldsymbol{N = 5 6})$ & Intervention $(\mathbf{n = 3 8})$ & Control $(\mathbf{n = 1 8})$ \\
\hline Child gender, male: $n(\%)$ & $40(71.43)$ & $27(71.05)$ & $13(72.22)$ \\
Child age, months: $M(S D)$ & $57.38(19.12)$ & $58.79(19.33)$ & $54.39(18.84)$ \\
Parent gender, female: $n(\%)$ & $55(98.21)$ & $37(97.37)$ & $18(100.0)$ \\
Parent age, years: $M(S D)$ & $33.59(6.67)$ & $34.13(7.07)$ & $32.44(5.75)$ \\
Parent age birth first child, & $26.16(5.97)$ & $26.30(6.53)$ & $25.89(4.76)$ \\
years, $M(S D)$ & & & \\
Post 16 education: $n(\%)$ & $42(75.0)$ & $27(71.05)$ & $15(83.33)$ \\
Married or in a stable & $46(82.14)$ & $30(78.95)$ & $16(88.89)$ \\
relationship: $n(\%)$ & & & \\
Employment: $n(\%)$ & $50(89.29)$ & $35(92.11)$ & $15(83.33)$ \\
Large family: $n(\%)$ & $15(26.79)$ & $12(31.58)$ & $3(16.67)$ \\
Teenage parent: $n(\%)$ & $8(14.29)$ & $5(13.16)$ & $3(16.67)$ \\
Attended a parenting & $16(28.57)$ & $11(28.95)$ & $5(22.78)$ \\
course $n(\%)$ & & & \\
\hline
\end{tabular}


Table 2. Baseline descriptive statistics $(N=56$; intervention $n=38$, control $n=18)$

\begin{tabular}{|c|c|c|c|c|}
\hline $\begin{array}{l}\text { Baseline observational } \\
\text { scores }\end{array}$ & $\begin{array}{l}\text { Intervention } \\
\text { Median } \\
\text { (range) }\end{array}$ & $\begin{array}{l}\text { Above CO } \\
n(\%)\end{array}$ & $\begin{array}{l}\text { Control } \\
\text { Median } \\
\text { (range) }\end{array}$ & $\begin{array}{l}\text { Above CO } \\
n(\%)\end{array}$ \\
\hline Observed Direct Command & $5.00(2-20)$ & l & $5.00(1-12)$ & l \\
\hline Observed Praise & $9.00(1-21)$ & / & $8.00(1-29)$ & I \\
\hline Observed Indirect Command & $34.00(7-98)$ & / & $18.00(5-79)$ & l \\
\hline Observed Questions & $74.00(22-192)$ & I & $74.00(3-141)$ & I \\
\hline Observed Negative Parenting & $10.00(1-39)$ & l & $9.00(0-26)$ & I \\
\hline Baseline scores (CO) & $\begin{array}{l}\text { Median } \\
\text { (range) }\end{array}$ & $\begin{array}{l}\text { Above CO } \\
n(\%)\end{array}$ & $\begin{array}{l}\text { Median } \\
\text { (range) }\end{array}$ & $\begin{array}{l}\text { Above CO } \\
n(\%)\end{array}$ \\
\hline ECBI Intensity (131) & $130.76(30.2)$ & $16(42.1)$ & $140.33(29.7)$ & $9(50.0)$ \\
\hline ECBI Problem (15) & $13.11(7.7)$ & $17(44.7)$ & $13.06(8.5)$ & $10(55.6)$ \\
\hline GHQ & $2.00(0-23)$ & $19(50)$ & $1.00(0-13)$ & $6(33)$ \\
\hline PSoC Total & $58.32(7.19)$ & I & $58.22(6.23)$ & I \\
\hline PSoC Efficacy & $24.16(3.67)$ & I & $23.50(3.20)$ & I \\
\hline PSoC Satisfaction & $29.76(4.96)$ & I & $30.28(4.87)$ & I \\
\hline Baseline parenting scores & $\mathbf{M}(S D)$ & Arnold CO & $\mathbf{M}(S D)$ & Arnold CO \\
\hline PS Laxness & $2.95(0.84)$ & 2.40 & $3.22(0.89)$ & 2.40 \\
\hline PS Over-reactivity & $2.84(0.71)$ & 2.40 & $2.76(0.74)$ & 2.40 \\
\hline PS Verbosity & $3.73(0.70)$ & 3.10 & $3.89(0.55)$ & 3.10 \\
\hline PS Total & $3.15(0.56)$ & 2.60 & $3.21(0.41)$ & 2.60 \\
\hline
\end{tabular}

Note: $\mathrm{CO}$ - cut-off; Arnold CO - mean score for a sample of parents of clinic referred children; ECBI - Eyberg Child Behaviour Inventory; PS - Parenting Scale; PSoC - Parent Sense of Competence; GHQ - General Health Questionnaire 


\section{Outcome Measures}

Table 3. Complete case results adjusted for baseline scores

\begin{tabular}{|c|c|c|c|c|c|c|c|}
\hline \multirow[t]{3}{*}{ Observational outcomes } & \multicolumn{2}{|l|}{ Intervention } & \multicolumn{2}{|l|}{ Control } & \multirow[t]{3}{*}{$F$} & \multirow[t]{3}{*}{$p$} & \multirow[t]{3}{*}{$d$} \\
\hline & Baseline $(n=38)$ & Follow-up $(n=25)$ & Baseline $(n=18)$ & Follow-up $(n=11)$ & & & \\
\hline & Median (range) & Median (range) & Median (range) & Median (range) & & & \\
\hline Observed Direct Command & $5.00(2-20)$ & $3.00(0-15)$ & $5.00(0-12)$ & $2.00(0-16)$ & 0.36 & .551 & 0.33 \\
\hline Observed Praise & $9.00(1-21)$ & $13.00(0-48)$ & $8.00(1-29)$ & $5.00(0-50)$ & 2.19 & .148 & 0.82 \\
\hline Observed Indirect Command & $34.00(7-98)$ & $20.00(4-64)$ & $18.00(5-79)$ & $27.00(18-90)$ & 6.36 & $.017 *$ & 0.59 \\
\hline Observed Questions & $74.00(22-192)$ & $58.00(9-136)$ & $74.00(3-141)$ & $38.00(8-109)$ & 0.43 & .517 & 0.23 \\
\hline Observed Negative Parenting & $10.00(1-39)$ & $3.00(0-30)$ & $9.00(0-26)$ & $6.00(0-29)$ & 0.69 & .411 & 0.32 \\
\hline \multicolumn{8}{|l|}{ Secondary outcomes } \\
\hline \multirow[t]{2}{*}{ GHQ } & $2.00(0-23)$ & $2.00(0-29)$ & $1.00(0-13)$ & $1.00(0-11)$ & 0.52 & .476 & 0.39 \\
\hline & $\mathbf{M}(S D)$ & $\mathbf{M}(S D)$ & $\mathbf{M}(S D)$ & $\mathbf{M}(S D)$ & & & \\
\hline ECBI Intensity (131) & $130.76(30.20)$ & $118.64(31.01)$ & $140.33(29.70)$ & $124.73(33.39)$ & 0.13 & .726 & 0.20 \\
\hline ECBI Problem (15) & $13.11(7.74)$ & $9.20(8.62)$ & $13.06(8.50)$ & $13.36(9.56)$ & 0.85 & .364 & 0.51 \\
\hline PS Total & $3.16(0.56)$ & $2.99(0.65)$ & $3.22(0.41)$ & $2.95(0.48)$ & 0.08 & .784 & 0.08 \\
\hline PS Laxness & $2.95(0.84)$ & $2.79(0.92)$ & $3.22(0.89)$ & $2.60(0.50)$ & 0.21 & .650 & 0.22 \\
\hline PS Over-reactivity & $2.84(0.71)$ & $2.69(0.76)$ & $2.76(0.74)$ & $2.82(0.95)$ & 1.20 & .282 & 0.18 \\
\hline PS Verbosity & $3.73(0.70)$ & $3.57(0.94)$ & $3.89(0.55)$ & $3.58(0.61)$ & 0.01 & .923 & 0.02 \\
\hline PSoC Total & $58.32(7.19)$ & $58.80(6.31)$ & $58.22(6.23)$ & $59.00(6.59)$ & 0.04 & .837 & 0.03 \\
\hline
\end{tabular}

Note: ECBI-Eyberg Child Behaviour Inventory; PS - Parenting Scale; PSoC - Parent Sense of Competence; GHQ - General Health Questionnaire; *Significant 
Table 4

Short-term maintenance effects for all outcomes measures for intervention group only $(n=20)$

\begin{tabular}{|c|c|c|c|c|c|}
\hline Observational outcomes & $\begin{array}{l}\text { Baseline } \\
M(S D) \\
(n=38)\end{array}$ & $\begin{array}{l}\text { 3-month f-up } \\
M(S D) \\
(n=25)\end{array}$ & $\begin{array}{l}\text { 6-month f-up } \\
M(S D) \\
(n=20)\end{array}$ & $\begin{array}{l}\text { Bl-6-month } \\
\text { Mean difference } \\
(95 \% \mathrm{CI})\end{array}$ & $\begin{array}{l}\text { 3-month - 6-month } \\
\text { Mean difference } \\
(95 \% \mathrm{CI})\end{array}$ \\
\hline Observed Direct Command & $5.00(2-20)$ & $3.00(0-15)$ & $5.00(0-19)$ & $0.04(-0.71,0.79)$ & $-0.32(-0.98,0.32)$ \\
\hline Observed Praise & $9.00(1-21)$ & $13.00(0-48)$ & $18.00(0-41)$ & $-1.41 *(-2.08,0.74)$ & $-0.55(-1.11,0.01)$ \\
\hline Observed Indirect Command & $34.00(7-98)$ & $20.00(4-65)$ & $28.00(4-37)$ & $1.54 *(0.68,2.41)$ & $-0.07(-0.69,0.55)$ \\
\hline Observed Questions & $74.00(22-192)$ & $58.00(9-136)$ & $64.00(7-136)$ & $0.81(-0.30,1.91)$ & $0.27(-0.48,1.03)$ \\
\hline Observed Negative Parenting & $10.00(1-39)$ & $3.00(0-30)$ & $3.00(1-9)$ & $1.09 *(0.50,1.69)$ & $-0.04(-0.60,0.52)$ \\
\hline \multicolumn{6}{|l|}{ Secondary outcomes } \\
\hline \multirow[t]{2}{*}{ GHQ } & $2.00(0-23)$ & $2.00(0-29)$ & $0.00(0-23)$ & $0.62(-0.19,1.43)$ & $0.27(-0.39,0.92)$ \\
\hline & $\mathbf{M}(S D)$ & $\mathbf{M}(S D)$ & $\mathbf{M}(S D)$ & & \\
\hline ECBI Intensity (131) & $130.76(30.20)$ & $118.64(31.01)$ & $115.45(31.44)$ & $13.70 *(5.25,22.15)$ & $2.70(-5.68,11.08)$ \\
\hline ECBI Problem (15) & $13.11(7.74)$ & $9.20(8.62)$ & $6.80(8.15)$ & $5.20 *(2.45,7.95)$ & $2.25 *(0.37,4.13)$ \\
\hline PS Total & $3.15(0.56)$ & $2.99(0.65)$ & $2.79(0.55)$ & $0.46^{*}(0.21,0.70)$ & $0.15(-0.05,0.35)$ \\
\hline PS Laxness & $2.95(0.84)$ & $2.79(0.92)$ & $2.61(0.76)$ & $0.47 *(0.18,0.77)$ & $0.18(-0.04,0.40)$ \\
\hline PS Over-reactivity & $2.84(0.71)$ & $2.69(0.76)$ & $2.45(0.56)$ & $0.55^{*}(0.13,0.97)$ & $0.27(-0.09,0.63)$ \\
\hline PS Verbosity & $3.73(0.70)$ & $3.57(0.94)$ & $3.24(0.88)$ & $0.56^{*}(0.26,0.86)$ & $-0.57 *(-1.04,-0.01)$ \\
\hline PSoC Total & $58.32(7.19)$ & $58.80(6.31)$ & $61.05(7.16)$ & $-3.80(-8.32,0.72)$ & $-1.85(-4.53,0.83)$ \\
\hline
\end{tabular}

Note: ECBI - Eyberg Child Behaviour Inventory; PS - Parenting Scale; PSoC - Parent Sense of Competence; GHQ - General Health

Questionnaire* Significant; CI-Confidence Intervals 


\section{Adverse Events}

There were no adverse events associated with this trial. 
